\title{
Enzyme-based amperometric galactose biosensors: a review
}

\author{
Prosper Kanyong $^{1,2}$ (D) Francis D. Krampa ${ }^{1,3} \cdot$ Yaw Aniweh $^{1} \cdot$ Gordon A. Awandare $^{1,3}$
}

Received: 26 May 2017 / Accepted: 14 August 2017 / Published online: 25 August 2017

(C) The Author(s) 2017. This article is an open access publication

\begin{abstract}
This review (with 35 references) summarizes the various strategies used in biosensors for galactose, and their analytical performance. A brief comparison of the enzyme immobilization methods employed and the analytical performance characteristics of a range of galactose biosensors are first summarized in tabular form and then described in detail. Selected examples have been included to demonstrate the various applications of these biosensors to real samples. Following an introduction into the field that covers the significance of sensing galactose in various fields, the review covers biosensors based on the use of galactose oxidase, with a discussion of methods for their immobilization (via crosslinking, adsorption, covalent bonding and entrapment). This is followed by a short section on biosensors based on the use of galactose dehydrogenase. The conclusion section summarizes the state of the art and addresses current challenges.
\end{abstract}

Keywords Galactose oxidase $\cdot$ Electrochemistry $\cdot$ Enzyme immobilization · Modified electrodes $\cdot$ Hydrogen peroxide . Glucose $\cdot$ Redox mediators $\cdot$ Screen-printed electrodes · Disposable sensors $\cdot$ Galactose dehydrogenase

Prosper Kanyong

p.kanyong@waccbip.org

1 West African Centre for Cell Biology of Infectious Pathogens (WACCBIP), University of Ghana, Legon, Accra, Ghana

2 School of Engineering, Ulster University, Jordanstown BT37 0QB, UK

3 Department of Biochemistry, Cell \& Molecular Biology, University of Ghana, Legon, Accra, Ghana

\section{Introduction}

The quantitative determination of galactose is of great importance in clinical chemistry, food and fermentation industries. There are two general analytical methods for the analysis of galactose in real samples, namely, separation by liquid or gas chromatography, and enzyme-based methods using galactose oxidase (GalOx) or galactose dehydrogenase (GADH) in conjunction with spectrophotometric, polarimetric, and fluorometric detection of enzymatic products [1,2]. Several commercial assay kits based on GADH have been developed. However, these methods are usually cumbersome and expensive, time-consuming and often require skilled personnel to operate them [1-3]. Electrochemical measurements, especially in conjunction with the use of biosensors, are most attractive for galactose analysis because they are rapid, relatively less expensive and sensitive and does not require pre-separation/treatment procedures $[2,3]$. This review discusses amperometric biosensors constructed based on GalOx or GADH, the method of enzyme immobilization, and where applicable, the application of the sensors to the analysis of real samples. To the best of our knowledge, this is the first time galactose biosensors have been reviewed.

\section{Galactose oxidase-based biosensors}

Galactose oxidase $(\mathrm{GalOx})$ is a free radical enzyme in which the active site contains five free radical ligands; namely two tyrosines, two histidines and a solvent molecule that is usually water. These free radicals are covalently linked to a copper center (Scheme 1) [4].

The electrochemical, biochemical and structural characteristics of GalOx has been extensively reviewed and discussed elsewhere [4, 5]. GalOx catalyzes the stereospecific oxidation 


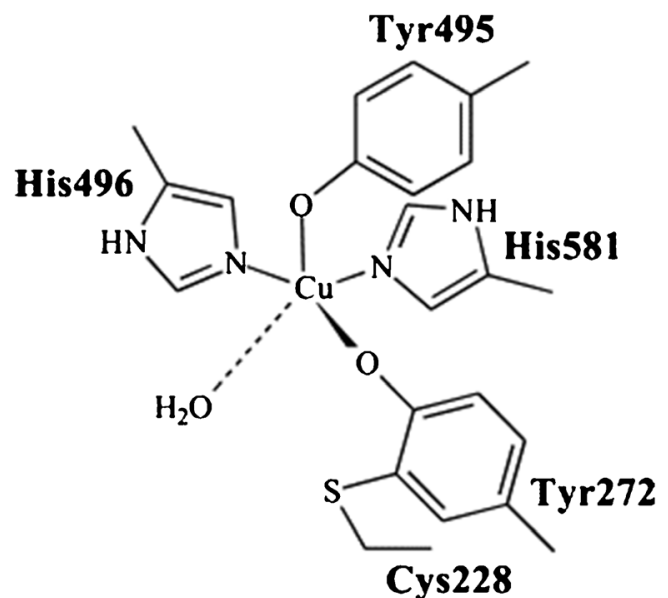

Scheme 1 Active site of galactose oxidase showing coordinating ligands (Reprinted from [4] with permission from Elsevier)

of $D$-isomers of a range of primary alcohol substrates such as $D$-galactose, dihydroxyacetone (DHA) as well as polysaccharides with $D$-galactose at their reducing end $[4,5]$.

Good biosensor selectivity is based on the enzyme catalysis of a substrate; in this case GalOx can be utilized to catalyze galactose. The electrochemical response process can be described by the following equation (Eq. 1):

$\alpha-\mathrm{D}-$ galactose $+\mathrm{O}_{2} \stackrel{\mathrm{GalOx}}{\longrightarrow} \alpha-\mathrm{D}$-galactohexodialdose $+\mathrm{H}_{2} \mathrm{O}_{2}(1)$

The electrochemical monitoring of the enzymatically produced $\mathrm{H}_{2} \mathrm{O}_{2}$ at an appropriate transducer generates the analytical response $[2,6]$. The amperometric monitoring of $\mathrm{H}_{2} \mathrm{O}_{2}$ can be done either via its oxidation (anodic response) or reduction (cathodic response) at the surface of an electrode. Electrodes modified with metal nanoparticles, metal oxides, graphene and graphene oxides and ionic liquids have been employed during the analysis of $\mathrm{H}_{2} \mathrm{O}_{2}[6,7]$. The anodic and cathodic behavior of $\mathrm{H}_{2} \mathrm{O}_{2}$ can be described by Eqs. 2 and 3, respectively;

$\mathrm{H}_{2} \mathrm{O}_{2} \rightarrow \mathrm{O}_{2}+2 \mathrm{H}^{+}+2 \mathrm{e}^{-}$

$\mathrm{H}_{2} \mathrm{O}_{2}+2 \mathrm{e}^{-}+2 \mathrm{H}^{+} \rightarrow 2 \mathrm{H}_{2} \mathrm{O}$

Typically, the use of redox mediators within the working electrode can allow $\mathrm{H}_{2} \mathrm{O}_{2}$ to be detected at low potentials $[2,3,6]$; thus, substantially reducing the effect of common interfering species in real samples. These favorable aspects of enzyme-based electrochemical biosensors would allow for the development of a rapid, selective and low-cost analytical devices; clearly, these are required for point-of-care applications.

\section{Cross-linking}

The first electrochemical method for quantitative galactose determination in biological fluids was reported by Taylor and his co-workers in 1977. A rapid, precise and accurate galactose sensing micro-method was developed by incorporating GalOx into glutaraldehyde (Glu). The GalOx-Glu mixture was subsequently casted onto a polycarbonate membrane (PC) and allowed to air dry [8]. The GalOxGlu-PC system was then used to modify the Model 23A Glucose Analyzer and employed for the analysis of galactose in both plasma and whole blood. Of the 39 compounds screened, the only physiologically relevant interference found was glycerone. The biosensor detected galactose up to $28.0 \mathrm{mM}$, however, the lower limit of detection was not stated. The detection of physiologically relevant species in complex matrices such as urine and whole blood using traditional electrode materials always present a challenging and significant problem with regards to the effect of interferences. In view of this, Manowitz and his coworkers [9] developed a biosensor that employed a composite polymer, with the ability to eliminate interferences. The system was used in conjunction with a flow injection analysis (FIA) system to detect galactose in human blood. To do this, a platinized carbon electrode was modified with a polymer film via the electropolymerization of 1,3diaminobenzene/resorcinol monomers. Thereafter, the polymer modified-platinized carbon electrode was coated with $1.25 \%$ Glu followed by aliquots of GalOx solution. After allowing the electrode to air dry, a second layer of Glu was drop-casted to form a sandwich format sensor. The sensor showed improved selectivity to $\mathrm{H}_{2} \mathrm{O}_{2}$ and minimized the effect of common interferences such as ascorbic acid, urate and paracetamol. When the biosensor was employed for the analysis of galactose in human plasma, the linearity was found to be up to $6.0 \mathrm{mM}$ with a lower limit of detection of $50.0 \mu \mathrm{M}$ and a storage stability of well over 30 days at room temperature [9].

Wang et al., also utilized Glu as a linker for immobilizing GalOx onto the surface of a Pt disc electrode pre-coated with electrodeposited Chitosan/Prussian Blue (PB) thin films [10]. The final biosensor was then used to detect galactose in human blood serum and fermentation broth. The response time of the sensor to galactose was found to be in the range of 42 $60 \mathrm{~s}$ with a sensitivity of $49.0 \mathrm{nA} \cdot \mathrm{mM}^{-1}$. It was found to be stable up to 30 days at $25{ }^{\circ} \mathrm{C}$ with a precision for repeated measurements of $3.4 \%$. The effect of interferences on the biosensor response was not evaluated. However, another study utilizing Glu as a cross-linker for immobilizing GalOx, based on the transduction capabilities of multi-walled carbon nanotubes (MWCNTs) and graphene composites, significantly eliminated the effect of interferences [11]. The first sensor was based on graphene, $\mathrm{Co}_{3} \mathrm{O}_{4}$ nanoparticles and chitosan 
composite modified glassy carbon electrode (GCE) while the second electrode was made from a composite of MWCNTs, $\mathrm{Co}_{3} \mathrm{O}_{4}$ nanoparticles and chitosan. In both cases, GalOx was immobilized onto the modified GCE surface by cross-linking with Glu. When the biosensors were applied to the analysis of galactose in human serum samples, the MWCNTs-based biosensor was found to have over 1.6-fold increase in sensitivity with a linearity range and detection limit far more superior to the graphene-based one. The electroanalytical performance of the two galactose biosensors are summarized in Table 1.

\section{Adsorption}

Buffone et al., 1980 physically adsorbed GalOx from the fungus Dactylium dendroides onto the Model 23A electrode. The immobilized GalOx/electrode system was thoroughly evaluated and found to be suitable for monitoring the treatment of infants with galactosemia, because it required very small sample volumes ( $\sim 25.0 \mu \mathrm{L}$ of serum) and was completed within a few minutes. It also provided analytical recoveries below $5.6 \mathrm{mM}$ [12]. However, the analytical performance characteristics such as sensitivity, linear range and detection limit was not stated. Ji and Zhang (1993) immobilized GalOx onto a platinized GCE pre-coated with Nafion [13]. The presence of Nafion, a permeation-selective membrane, in the sensor design helped reduce the effect of interfering electroactive species such as uric acid and ascorbic acid. The biosensor exhibited a linear range of $0.25-4.25 \mathrm{mM}$ and a response time of $30 \mathrm{~s}$. There were no obvious variations in the sensor response for 300 repeated measurements in 10 days. Miyata et al., 1995 constructed a micro-sensor by adsorbing GalOx onto a porous carbon electrode pre-coated with a tris(2,2'-bipyridine) complex of osmium (II/III) as a redox mediator and utilized it for the detection of galactose [14]. The amperometric response of

Table 1 Analytical performance characteristics of galactose oxidase-based amperometric biosensors

\begin{tabular}{|c|c|c|c|c|c|c|c|}
\hline Sensor design & Sensitivity & $\begin{array}{l}\text { LOD } \\
(\mu \mathrm{M})\end{array}$ & $\begin{array}{l}\text { Linear range } \\
(\mathrm{mM})\end{array}$ & $\begin{array}{l}\text { Response } \\
\text { time }(\mathrm{s})\end{array}$ & $\begin{array}{l}\text { Storage } \\
\text { stability } \\
(\text { days }) / \\
\mathrm{K}_{\mathrm{m}}(\mathrm{mM})\end{array}$ & Real sample & Ref \\
\hline GalOx/Ferrocene/Graphite & $0.9 \mathrm{~mA} \cdot \mathrm{M}^{-1}$ & 2.8 & $5.6 \times 10^{-3}-0.6$ & 44 & $52 /-$ & - & {$[1]$} \\
\hline $\mathrm{GalOx} / \mathrm{CA} / \mathrm{Co}-\mathrm{SPCE}$ & $7.0 \mu \mathrm{A} \cdot \mathrm{mM}^{-1} . \mathrm{cm}^{-2}$ & 20.0 & $0.1-25.0$ & 30 & $14 / \sim 12.5$ & Serum & [2] \\
\hline $\mathrm{GalOx} / \mathrm{CA} / \mathrm{Co}-\mathrm{SPCE}$ & $3.1 \mu \mathrm{A} \cdot \mathrm{mM}^{-1} \cdot \mathrm{cm}^{-2}$ & 0.2 & $1.98-9.25$ & - & $14 /-$ & $\begin{array}{l}\text { Culture } \\
\text { medium }\end{array}$ & {$[3]$} \\
\hline $\mathrm{GalOx} / \mathrm{Glu} / \mathrm{PC} / \mathrm{H}_{2} \mathrm{O}_{2}$ electrode & - & - & $0.0-28.0$ & 40 & $7 /-$ & Plasma, Blood & {$[8]$} \\
\hline $\mathrm{Glu} / \mathrm{GalOx} / \mathrm{Glu} / 1,3-\mathrm{DAB} / \mathrm{Res} / \mathrm{Pt}$ & - & 50.0 & $50 \times 10^{-3}-6.0$ & 18 & $30 / 2.5$ & Plasma & [9] \\
\hline $\mathrm{GalOx} / \mathrm{Glu} / \mathrm{Chit} / \mathrm{PB} / \mathrm{Pt}$ & 49.0 nA. $\mathrm{mM}^{-1}$ & 60.0 & $0.1-6.0$ & $42-60$ & $30 /-$ & Serum & [10] \\
\hline $\mathrm{GalOx} / \mathrm{Co}_{3} \mathrm{O}_{4} /$ Graphene/GCE & $6.6 \mu \mathrm{A} \cdot \mathrm{mM}^{-1} \cdot \mathrm{cm}^{-2}$ & 3.0 & $9.0 \times 10^{-3}-0.6$ & 15 & $\sim 30 / 1.2$ & Serum & [11] \\
\hline $\mathrm{GalOx} / \mathrm{Co}_{3} \mathrm{O}_{4} / \mathrm{MWCNTs} / \mathrm{GCE}$ & $10.4 \mu \mathrm{A} \cdot \mathrm{mM}^{-1} \cdot \mathrm{cm}^{-2}$ & 0.9 & $9.0 \times 10^{-3}-1.0$ & 20 & $\sim 30 / 0.7$ & Serum & [11] \\
\hline $\mathrm{GalOx} / \mathrm{Nafion} / \mathrm{Pt}$ & 100.0 nA.mM ${ }^{-1}$ & - & $0.25-4.25$ & $<30$ & $10 /-$ & Serum & [13] \\
\hline $\mathrm{GalOx} / \mathrm{PVF} / \mathrm{O}_{2}$ electrode & - & $2.8 \times 10^{4}$ & $28.0-168.0$ & - & $90 /-$ & Milk & [17] \\
\hline GalOx/Collagen $/ \mathrm{H}_{2} \mathrm{O}_{2}$ electrode & $1.0-3.0 \mathrm{~mA} \cdot \mathrm{M}^{-1}$ & 0.5 & $5.0 \times 10^{-4}-0.6$ & 60 & $\sim 300 /-$ & Serum & [19] \\
\hline $\mathrm{PC} / \mathrm{GalOx} /$ Ferrocinium/CPE & - & - & - & $<120$ & $-/ 14.5$ & - & [21] \\
\hline $\mathrm{PU} / \mathrm{GalOx} /$ Polyacrylamide $/ \mathrm{O}_{2} /$ electrode & $15.0-51.0$ pA.mM ${ }^{-1}$ & 2.0 & up to $1.5 / 10.0$ & $0.5-5.0$ & $30 /-$ & - & [21] \\
\hline GalOx/P3HT/SA/ITO & $4.2 \mathrm{nA} \cdot \mathrm{mM}^{-1}$ & $5.6 \times 10^{-3}$ & $5.6-22.2$ & 6 & $90 /-$ & Milk & [22] \\
\hline GalOx/PEG/Polyanion/Pt & $106.0 \mathrm{nA} \cdot \mathrm{mM}^{-1} \cdot \mathrm{cm}^{-2}$ & - & $0.0-24.0$ & $<40$ & $-/ 43.0$ & - & [23] \\
\hline GalOx/Glu/Chit/SWCNT-GCE & 1126.0 nA. $\mathrm{Mm}^{-1}$ & 25.0 & up to 1.0 & - & $-/-$ & Blood & [24] \\
\hline GalOx/Poly-GMA-Co-VFc/Pt & 23.0 nA. $\mathrm{mM}^{-1} \cdot \mathrm{cm}^{-2}$ & 100.0 & $2.0-20.0$ & 5 & $\sim 30 / 16.0$ & - & [25] \\
\hline $\mathrm{GalOx} / \mathrm{PEP} / \mathrm{Au}$ & $1.8 \mu \mathrm{A} \cdot \mathrm{mM}^{-1}$ & 25.0 & $2.0-16.0$ & 5 & $\sim 10 / 14.7$ & - & [26] \\
\hline GalOx/Microtubules/ITO & $\sim 6.4 \mu \mathrm{A} \cdot \mathrm{mM}^{-1} \cdot \mathrm{cm}^{-2}$ & 10.0 & $0.1-1.0$ & $30-40$ & $7 /-$ & - & [27] \\
\hline GalOx/Polypyrrole/Pt & $3.5-14.7 \mathrm{~mA} \cdot \mathrm{M}^{-1} \cdot \mathrm{cm}^{-2}$ & 500.0 & $5.0 \times 10^{-4}-2.0$ & - & $15 / 1.0-4.0$ & Blood & [28] \\
\hline GalOx/Polypyrrole/Pt & 37.5 nA.mM ${ }^{-1}$ & - & up to 2.0 & 30 & $-/ 15.8$ & - & [29] \\
\hline $\mathrm{GalOx} / \mathrm{PVF} / \mathrm{Pt}$ & $\sim 1.0 \mu \mathrm{A} \cdot \mathrm{mM}^{-1}$ & - & up to 40.0 & $30-40$ & $3 / 21.7$ & - & [30] \\
\hline GalOx/Polypyrrole-[p(HEMA)]/Pt & $937.0 \mu$ A.M $\mathrm{M}^{-1}$ & 25.0 & $5.0 \times 10^{-2}-10.0$ & 70 & $270 /-$ & Serum & [31] \\
\hline $\mathrm{GalOx} /$ Laponite clay/Pt & $85.0 \mathrm{~mA} \cdot \mathrm{M}^{-1} \cdot \mathrm{cm}^{-2}$ & 1.0 & $1.0 \times 10^{-3}-1.6$ & 5 & $\sim 28 /-$ & - & [33] \\
\hline $\mathrm{GADH} / \mathrm{NADP}^{+} / \mathrm{Os} / \mathrm{CPE}$ & $1.7 \mu \mathrm{A} \cdot \mathrm{mM}^{-1} . \mathrm{cm}^{-2}$ & $2.0 \times 10^{2}$ & $1.0-3.0$ & - & $-/ 68$ & Milk & [35] \\
\hline
\end{tabular}

Glu glutaraldehyde, $P C$ polymcarbonate membrane, $C P E$ carbon paste electrode, 1,3-DAB 1,3-diaminobenzene, Res resorcinol. $P U$ polyurethane, $P V F$ polyvinylferrocenium, [p(HEMA)] poly(2-hydroxyethyl methacrylate), $P 3 H T$ poly-3-hexyl thiophene. $S A$ stearic acid, ITO indium tin-oxide, $P$-4-MP Poly-4-methoxyphenol, $C P E$ cabron paste electrode, $O s$ osphendione, $G A D H$ galactose dehydrogenase, $P V F$ polyvinyl formal 
the biosensor to galactose was found to be linear over concentrations ranging from $10.0 \mathrm{mM}$ to $5.0 \times 10^{3} \mathrm{mM}$, with a correlation coefficient of 0.999 . However, these biosensors did not have prolonged stability.

In view of this, a galactose biosensor was fabricated by drop-coating GalOx on the surface of a graphite electrode adsorbed with the redox mediator, ferrocene from paraffin solution [1]. The storage stability of the biosensor was highly improved when the sensor was treated with both diethylaminoethyl (DEAE)-dextran and inositol. It exhibited a low limit of detection of $2.83 \mu \mathrm{M}$, a response time of $44.0 \mathrm{~s}$ and linearity range of 5.6-610.6 $\mu \mathrm{M}$. During repeated assays for over $5 \mathrm{~h}$, there was only $5 \%$ drop in the original response; thus, indicating that the biosensor was highly stable and suitable for routine analysis of galactose. Mannino, Cosio and Buratti, 1999 fabricated a sensitive, specific and rapid biosensor for measuring galactose content in dairy products [15]. The biosensing probe consisted of rhodium-on-carbon paste with adsorbed GalOx. The sensor exhibited enhanced selectivity (with discrimination against interferences such as ascorbic acid) and enzymatically produced $\mathrm{H}_{2} \mathrm{O}_{2}$ was measured at lower potentials. The biosensor response was linear from $1.4 \mathrm{mM}$ to $22.2 \mathrm{mM}$ with a limit of detection of $0.5 \mathrm{mM}$ [15]; these analytical performance characteristics demonstrated that the rhodium-on-carbon paste/GalOx electrode was a robust amperometric biosensor that can be employed for quality control in the dairy industry.

Jia et al., 2003 utilized thin-film technology to fabricate a microchip sensor strip consisting of a Pt disk as the working electrode [16]. Firstly, the Pt disk was coated with Nafion thin-film followed by GalOx. The presence of Nafion within the sensor design significantly enhanced its selectivity towards galactose in serum samples. The response time for galactose was $<40 \mathrm{~s}$ with a linearity range from $0.1 \mathrm{mM}$ to $8.0 \mathrm{mM}$. The precision for repeated measurements was $\sim 1.7 \%$ and the influence of common interferences such as ascorbic acid, uric acid and glucose did not cause any significant change to the analysis of galactose; this high selectivity for galactose was attributed to the ability of the Nafion membrane to remove interferences. To validate the sensor, it was employed to recover known concentrations of galactose spiked into serum samples and the recovery values ranged from $97.0 \%$ to $105.0 \%$; this demonstrated the suitability of the biosensor for routine analysis of galactose in real samples. Lastly, the biosensor retained $90.0 \%$ of its initial activity towards galactose following 1 month of storage. However, there was a 3 -fold increase in the stability of a similar biosensor developed by adsorbing GalOx onto polyvinyl fluoride (PVF) membrane to form GalOx/PVF system. The GalOx/PVF layer was then attached to an $\mathrm{O}_{2}$ electrode and used for the determination of galactose in milk and milk products [17]. The response of the bioelectrode to standard concentrations of galactose was found to be linear from $28.0 \mathrm{mM}$ to $168.0 \mathrm{mM}$ (the former being the limit of detection). However, the biosensor did not eliminate the effect of interferences during the analysis of real samples.

In another study [18], GalOx was physically adsorbed onto microporous polyacrylonitrile thin films. The biosensor appeared to have good stability and was highly reproducible. When the sensor was employed for the determination of galactose in human sera, the amperometric current responses increased linearly with increasing galactose concentrations from $20.0 \mu \mathrm{M}$ to $1.6 \mathrm{mM}$. Kanyong et al., 2013 developed a disposable amperometric biosensor (Fig. 1a) for the measurement of circulating galactose in infant serum [2]. The sensor designed comprised of a water-based screen-printed carbon electrode (SPCE), incorporating the redox mediator, cobalt phthalocyanine $(\mathrm{CoPc})$ (Co-SPCE). Firstly, Co-SPCE was covered with cellulose acetate (CA) followed by physical adsorption of GalOx (Fig. 1b). The operation of the biosensor was based on the interaction of galactose with the immobilized GalOx which leads to the production of $\mathrm{H}_{2} \mathrm{O}_{2}$; the $\mathrm{H}_{2} \mathrm{O}_{2}$ then diffuses through the pores of the $\mathrm{CA}$ membrane onto the underlying electrode where it undergoes a chemical oxidation by the $\mathrm{CoPc}\left(\mathrm{Co}^{2+}\right)$ to produce $\mathrm{CoPc}\left(\mathrm{Co}^{+}\right)$(Eq. 4):

$2 \mathrm{Co}^{2+} \mathrm{H}_{2} \mathrm{O}_{2} \rightarrow 2 \mathrm{Co}^{+}+\mathrm{O}_{2}+2 \mathrm{H}^{+}$

The electrochemical re-oxidation of $\mathrm{Co}^{+}$to $\mathrm{Co}^{2+}$ (Eq. 5) occurred at an applied voltage of $+0.5 \mathrm{~V}$ to produce the analytical response:

$\mathrm{Co}^{+} \rightarrow \mathrm{Co}^{2+}+e^{-}$

Clearly, the detection of galactose via this system has the advantage that the $\mathrm{H}_{2} \mathrm{O}_{2}$ will pass through the small pores of the CA membrane that would exclude interferences commonly present in human bodily fluids.

The effect of temperature, ionic buffer strength, enzyme loading and $\mathrm{pH}$ on the analytical performance characteristics of the biosensor was thoroughly optimized. To compensate for the influence of interferences in serum that was not eliminated via the use of the CA membrane, the researchers fabricated a dummy biosensor (Fig. 1c) by using the same mass of bovine serum albumin (BSA) as that of the enzyme. The effects of interferences from the serum were measured with the dummy biosensor and subtracted from that of the galactose biosensor response. Using the dummy biosensor in conjunction with the galactose biosensor, the influence of several interfering species such as acetaminophen, uric acid, urea and ascorbic acid was successfully compensated for. The amperometric response of the optimized galactose biosensor on exposure to standard concentrations of galactose was linear from $0.1 \mathrm{mM}$ to $25.0 \mathrm{mM}$ with a sensitivity of $7.0 \mu \mathrm{A} \cdot \mathrm{mM}^{-1} . \mathrm{cm}^{-2}$ and a 
Fig. 1 Design of a cobalt phthalocyanine screen-printed carbon electrode (Co-SPCE); b galactose biosensor and; c Dummy biosensor. RE:

Reference Electrode, WE:

Working Electrode

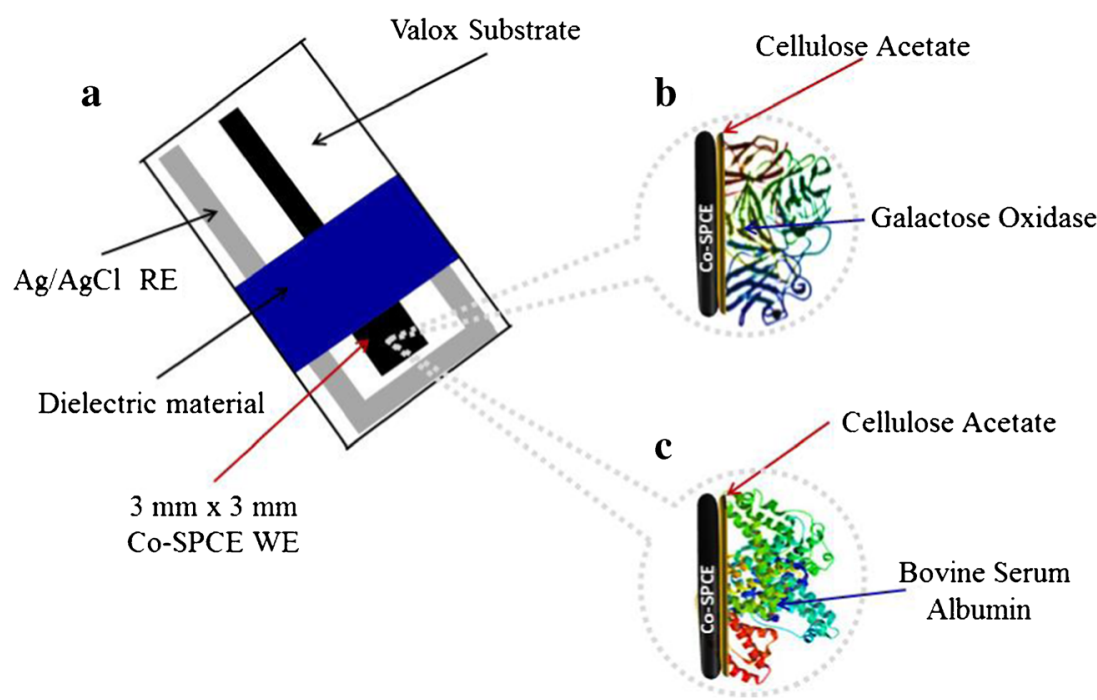

calculated limit of detection of $20.0 \mu \mathrm{M}$. When the biosensor was used to analyze serum samples, the precision obtained on unspiked and spiked serum $(n=6)$ were $1.10 \%$ and $0.11 \%$, respectively with a recovery of $99.9 \%$.

Using the same biosensor design, the researchers fabricated a microband galactose biosensor for real-time monitoring of the update of galactose by hepatocellular carcinoma cells after exposing the cells to various toxic compounds [3]. The biosensor maintained steady-state current responses in culture medium for batch-type analysis to monitor the depletion of galactose by hepatocytes in a cell density-dependent manner. It provided a sensitivity of $7.3 \mu \mathrm{A} . \mathrm{mM}^{-1} . \mathrm{cm}^{-2}$, a linear range up to $9.52 \mathrm{mM}$ and a coefficient of variation of $1.2 \%$.

\section{Covalent bonding}

A selective biosensor for galactose analysis was developed through covalent attachment of GalOx onto highlypolymerized collagen films [19]. An activated collagen film was immersed in GalOx solution at alkaline $\mathrm{pH}$; thus, the enzyme was covalently linked to the collagen by reaction with acyl azides. The enzyme/collagen film system was then attached to a Pt anode, whose potential was maintained at $+0.65 \mathrm{mV}$ vs. $\mathrm{Ag} / \mathrm{AgCl}$ reference electrode, and used for monitoring the enzyme-generated $\mathrm{H}_{2} \mathrm{O}_{2}$. To compensate for electrochemical interferences in biological fluids, a dummy electrode was used to record the interfering current. The response of the dummy electrode was then subtracted from the response of the enzyme-based electrode; thus, providing an accurate measurement of the enzymatically generated $\mathrm{H}_{2} \mathrm{O}_{2}$. A steady-state response was obtained within $5 \mathrm{~min}$ and the biosensor exhibited a calibration linearity from $0.5 \mu \mathrm{M}$ to $600.0 \mu \mathrm{M}$ and depending on the enzyme membrane activity, a sensitivity in the range of $1.0-3.0 \mathrm{~mA}^{-1} \mathrm{M}^{-1}$ was found.
Galactose is an important substrate for fermentation and food production. Lundback and Olsson reported an amperometric technique for the detection of galactose in such systems by immobilizing GalOx onto reactors [20]. The enzyme was immobilized via aryloamino-derivatized controlled-pore glass and packed into the reactors. The $\mathrm{H}_{2} \mathrm{O}_{2}$ detection was mediated via hexacyanoferrate (II). The response of the sensor to standard solutions of galactose was found to be linear from $2.0 \mu \mathrm{M}$ to $60.0 \mu \mathrm{M}$ with a throughput of 45 samples $/ \mathrm{h}$ and a relative standard deviation of $0.4 \%$. In another study, Peteu, Emerson and Worden (1996) covalently attached GalOx onto the tip of a Clark-type $\mathrm{O}_{2}$ microelectrode [21]. The enzyme was mixed with a homogenous solution of polyacrylamide and the tip of the electrode was held in contact with the solution for a few seconds and then withdrawn; this procedure was repeated several times until a small bulb $(\sim 40.0 \mu \mathrm{m})$ was formed at the tip of the electrode. The bulb was then allowed to air-dry. Using the same procedure, the tip of the electrode was coated with polyurethane membrane. The limit of detection of the sensor was found to be $2.0 \mu \mathrm{M}$ with a linear range that is dependent on the thickness of the polyurethane film but extended up to $10.0 \mathrm{mM}$. It also exhibited response times of $0.5-5.0 \mathrm{~s}$, a sensitivity of $51.0 \times 10^{-5} \mathrm{~mA} . \mathrm{M}^{-1}$ and a storage stability of up to 1 month [21].

Sharma et al., 2004 prepared an enzymatic amperometric biosensor for the determination of galactose in milk and milk products by immobilizing $\mathrm{GalOx}$ with poly-3-hexyl thiophene and with stearic acid onto an indium tin-oxide (ITO)-coated glass plate via Langmuir-Bloggett (LB) film deposition technique [22]. This design improved the shelf-life of the biosensor to well over 90 days at $40{ }^{\circ} \mathrm{C}$ storage conditions. The response time was $60 \mathrm{~s}$ with a linearity of 5.6-22.2 mM. In another study, galactose biosensors were fabricated by covalently attaching GalOx onto polyanion via polyethylene glycol (PEG) spacer (Fig. 2) [23]. 


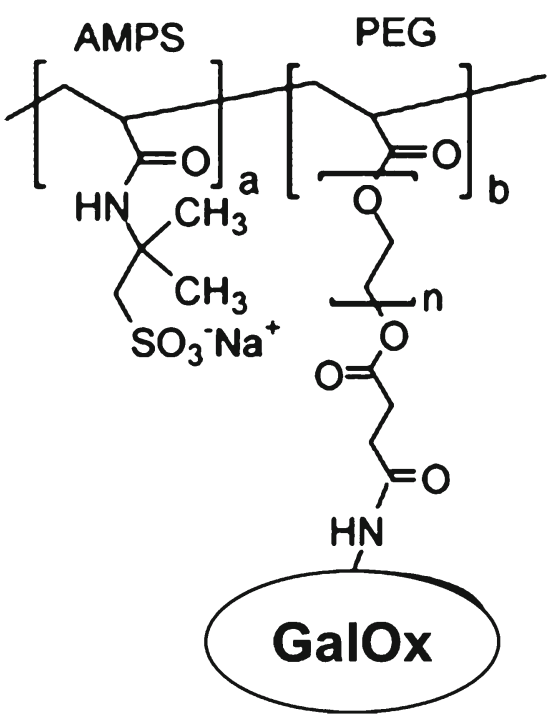

Fig. 2 Schematic representation of GalOx immobilization to form a polyanion/PEG/GalOx conjugate. AMPS; 2-Acrylamido-2methylpropane sulfonic acid (Polyanion) (Reprinted from [23] with permission from Elsevier)

The polyanion/PEG/GalOx conjugate was then entrapped within a polypyrrole film. This was done by electrodepositing onto a Pt electrode from a solution containing pyrrole and the polyanion/PEG/GalOx conjugated dopant; thus, the resulting polyanion/PEG/GalOx conjugate was effectively entrapped into the polypyrrole matrix. The amperometric response of the polypyrrole/polyanion/PEG/GalOx electrode to standard concentrations of galactose was found to be linear from $0.0 \mathrm{mM}$ to $24.0 \mathrm{mM}$ with a sensitivity of $106.0 \mathrm{nA} . \mathrm{mM}^{-}$ ${ }^{1} \mathrm{~cm}^{-2}$ and an improved response time of $\sim 40 \mathrm{~s}$.

Tkac et al., utilized chitosan, a natural polymer, to solubilize carbon nanotubes. Further stabilization of the chitosan/ carbon nanotube dispersion was done via chemical crosslinking with Glu. The free aldehyde groups of Glu were subsequently used to covalently immobilized GalOx [24]. To do this, a polished GCE was coated with the chitosan/carbon nanotube dispersion, followed by layers of Nafion, GalOx and Nafion, respectively. The use of the permeationselective membrane, Nafion, to sandwich GalOx led to an interference-free detection of galactose in the presence of high concentrations of uric acid and paracetamol. The biosensor was found to be robust, reliable and was successfully used to analysis galactose in blood plasma with sample throughput of $150 / \mathrm{h}$. The limit of detection was found to be $2.0 \mu \mathrm{M}$. When the biosensor was used in conjunction with amperometry and a Flow Injection Analysis (FIA) system to recover known concentrations of galactose spiked into blood plasma, the recovery index was found to be between 101.2 and $102.7 \%$, with a precision of $2.0 \%$. Another galactose biosensor was developed by covalently immobilizing GalOx onto a polymeric mediator, polyglycidyl methacrylate-co-vinyl ferrocene film via its amine groups (Fig. 3) [25].
Due the incorporation of the redox mediator, vinyl ferrocene, into the sensor design, $\mathrm{H}_{2} \mathrm{O}_{2}$ was measured at lower potentials and the final biosensor was found to have good stability, repeatability and reproducibility. The optimized sensor exhibited a linear response range of $2.0-20.0 \mathrm{mM}$, a limit of detection of $0.1 \mathrm{mM}$ and a response time of $5 \mathrm{~s}$. Similarly, a novel amperometric galactose biosensor was developed by immobilizing GalOx onto electrochemically synthesized poly(N-glycidylpyrrole-co-pyrrole) film via a one-step covalent attachment procedure and without the use of any coupling agents [26]. When the amperometric response of the biosensor was measured as a function of increasing concentrations of galactose, at an applied voltage of $+0.7 \mathrm{~V}$ vs $\mathrm{Ag} / \mathrm{AgCl}$ reference electrode, the response time was less than $5 \mathrm{~s}$. The linearity range was from $2.0 \mathrm{mM}$ to $16.0 \mathrm{mM}$ with a sensitivity of $1.75 \mu \mathrm{A} \cdot \mathrm{mM}^{-1}$. To enhance the sensitivity of galactose sensing, Lee et al., 2011 covalently attached galactose oxidase onto a bundle of conducting polymer microtubules [27]. The microtubules, prepared via the electropolymerization of 3,4ethylenedioxythiophene (EDOT) onto a template, had structures which provided larger enzyme encapsulating space per unit area of the sensing electrode compared to other immobilizing procedures. When the biosensor was employed for the analysis of galactose, it exhibited a linear behavior from $0.1 \mathrm{mM}$ up to $1.0 \mathrm{mM}$ galactose with a sensitivity of $6.37 \mu \mathrm{A} . \mathrm{mM}^{-1} \cdot \mathrm{cm}^{-2}$, a limit of detection of $10.0 \mu \mathrm{M}$ (based on $3 \times$ the baseline noise) and a response time of $30-40 \mathrm{~s}$.

\section{Entrapment}

This method involves integrating an enzyme within the lattice of an appropriate polymer matrix or membrane. In addition to the immobilization of enzymes, membranes can also eliminate the effect of interfering electroactive species that may be present in real samples such as blood or urine [2,3]. A series of amphiphilic pyrrolylalkylammonium ions differing in the size of their ammonium heads were used as a matrix for the immobilization of GalOx onto Pt and carbon disk electrodes [28]. Polypyrrolic films were electrogenerated from adsorbed amphiphilic pyrrole-galactose oxidase mixtures. The enzyme retention properties of the polymers were investigated and it showed that the lesser the hydrophobicity of the film, the higher the enzyme immobilization efficiency and the sensitivity of the biosensor was related to the permeability of the polymer. Compared to classical electrochemical enzyme entrapments, the method offered the possibility of controlling the composition of the enzyme-polymer film and allows for the determination of the amount of the immobilized enzyme; thus, its specific activity. There was no decrease in the enzymatic activity of the biosensor for 
Fig. 3 Structure of polyglycidyl methacrylate-co-vinyl ferrocene (Poly-GMA-co-VFc) and covalent binding of GalOx to the Poly-GMA- co-VFc film electrode (Redrawn from [25])

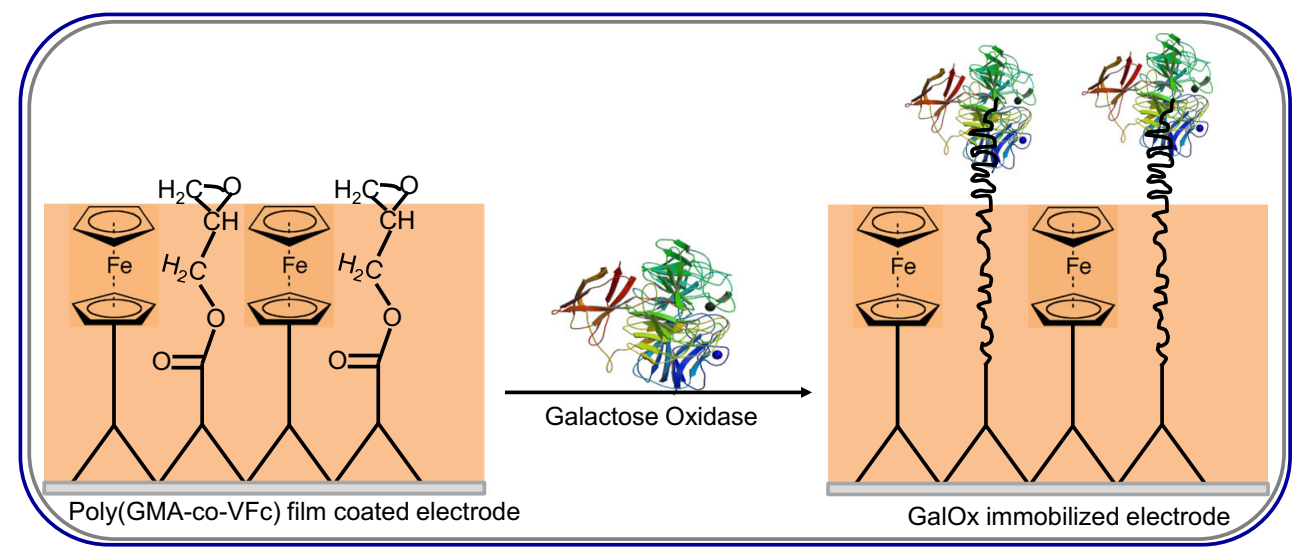

up to 15 days and sensitivity values in the range of $3.5-$ $14.7 \mathrm{~mA} \cdot \mathrm{M}^{-1} . \mathrm{cm}^{-2}$ were found for the various polymers with entrapped GalOx. Even though the influence of interfering species such as uric acid, ascorbic acid and paracetamol were found to decrease markedly with increasing polymer hydrophobicity, the sensor design was unable to eliminate the effect of the interferences.

In addition to this, a bio-platinum electrode for galactose was developed via the electrochemical polymerization of pyrrole in a buffer solution containing GalOx, which became entrapped in the growing polymer film during the electropolymerization [29]. The experimental conditions for this study ensured that the particles of polypyrrole were arranged tightly during the formation of the film; the space between the particles were sufficiently larger than the diameter of the enzyme; thus, the enzyme was entrapped in the polymer film based on the doping principles of conducting polymers. A Raman spectrum of the polypyrrole GalOx biosensor showed a characteristic peak for an amino acid at $1414 \mathrm{~cm}^{-1}$; an evidence for successful entrapment of the GalOx within the polypyrrole film. When the bioelectrode was used to analyze galactose, its response current increased linearly with increasing concentration of galactose up to $2.0 \mathrm{mM}$ [29].

Gülce and co-workers [30] reported a Pt electrode coated with a redox polymer, polyvinylferrocenium $\left(\mathrm{PVF}^{+}\right)$, which catalyzed the oxidation and reduction of organic species as well as $\mathrm{H}_{2} \mathrm{O}_{2}$. The chemical oxidation of $\mathrm{H}_{2} \mathrm{O}_{2}$ by the redox polymer, $\mathrm{PVF}+$ occurred as (Eq. 6):

$$
2 \mathrm{PVF}^{+} \mathrm{H}_{2} \mathrm{O}_{2} \rightarrow 2 \mathrm{PVF}+\mathrm{O}_{2}+2 \mathrm{H}^{+}
$$

followed by the electro-regeneration of the PVF at an applied potential of $+0.7 \mathrm{~V}$ vs. SCE (Eq. 7):

$2 \mathrm{PVF} \rightarrow 2 \mathrm{PVF}^{+}+2 e^{-}$

The matrix was used as a pre-concentration agent for the detection of inorganic anions. Consequently, the anion exchange properties of the redox polymer were utilized for developing the bioelectrode for galactose by immobilizing GalOx onto the matrix. When the bioelectrode was used to analyze galactose, a response time of $30-40 \mathrm{~s}$ was found and the upper limit of the linear working portion was $40.0 \mathrm{mM}$; these performance characteristics were deemed to be satisfactory for the analysis of galactose in bodily fluids.

Brahim et al., 2002 developed a rapid, two-step protocol for constructing galactose biosensors by entrapping GalOx within a polymeric composite [31]. The polymeric composite was formed as an interpenetration network of polypyrrole grown within a UV cross-linked-poly-(2-hydroxyethyl methacrylate) hydrogel. The sensor exhibited a linear response range, calculated limit of detection and response time of $5.0 \times 10^{1}-1.0 \times 10^{4} \mu \mathrm{M}, 25.0 \mu \mathrm{M}$ and $70 \mathrm{~s}$, respectively. When the biosensor was employed with amperometry for the analysis of serum samples, the precision values obtained for same day and day-to-day analyses were $3.8 \%$ and $4.4 \%$, respectively, with recoveries ranging from $97.0 \%$ to $105.0 \%$. Moreover, the biosensor eliminated the influence of interferences such as urate, paracetamol and ascorbate and retained $70.0 \%$ of its initial activity after 9 months of storage in a desiccator at $4{ }^{\circ} \mathrm{C}$. Another polymer based-galactose oxidase sensor was fabricated via a one-step procedure via the electropolymerization of 4-methoxyphenol in the presence of GalOx onto a Pt electrode [32]. Polymeric thin films of $1.5 \mu \mathrm{m}$ thickness with GalOx entrapped were prepared and the biosensor had a linear response to increasing concentrations of galactose up to $16.0 \mathrm{mM}$ with a response time of $<4 \mathrm{~s}$. However, the sensor design did not eliminate the effect of interferences such as ascorbic acid, paracetamol and oxalic acid. The biosensor also lost well over $52 \%$ of its initial response to galactose within 30 days; thus, making it unsuitable for routine analysis of galactose.

An amperometric galactose biosensor was developed by entrapping GalOx within a laponite clay film coated onto a Pt electrode surface [33]. The laponite was found to provide a favorable environment for GalOx activity; consequently, the optimized sensor was found to respond linearly to increasing galactose concentrations from $1.0 \mu \mathrm{M}$ to $1.6 \mathrm{mM}$ 
(the former being detection limit) with a sensitivity of $\sim 85.0 \mathrm{~mA} \cdot \mathrm{M}^{-1} \cdot \mathrm{cm}^{-2}$. It was stable for up to 1 month at $4{ }^{\circ} \mathrm{C}$ and the response time was $\sim 5 \mathrm{~s}$. The effect of interferences on the biosensor response was not eliminated. In a similar study, GalOx was immobilized within the laponite clay matrix and the laponite-GalOx conjugate was deposited on screen-printed electrodes already modified with Prussian blue and poly-( $O$-phenylenediamine) [34]. Using the bioelectrode, the reduction of enzymatically generated $\mathrm{H}_{2} \mathrm{O}_{2}$ was performed at a significantly lower potential $(-0.2 \mathrm{~V}$ vs $\mathrm{Ag} / \mathrm{AgCl})$ and the limit of detection for galactose was found to be $13.0 \mu \mathrm{M}$ with a sensitivity of $16.0 \mathrm{~mA} \cdot \mathrm{M}^{-1} . \mathrm{cm}^{-2}$. Consequently, the laponite is deemed to be a suitable material for entrapping GalOx.

\section{Galactose dehydrogenase-based biosensors}

The determination of galactose in real samples has been traditionally achieved via the use of the enzyme galactose oxidase. However, Maestre et al., 2005 utilized a galactose dehydrogenase $(\mathrm{GADH})$ from thermoacidophilic archaebacterium [35]. GADH is a $\mathrm{NADP}^{+} / \mathrm{NADPH}$ dependent dehydrogenase having catalytic activity not only for galactose but also for other sugars such as glucose and xylose. In this study, pretreated graphite powder, osphendione (Os) (a redox mediator), $\mathrm{NADP}^{+}, \mathrm{GADH}$ and paraffin oil were thoroughly mixed and packed into a Teflon tube to form a carbon paste electrode. The catalytic and electrochemical reactions for galactose based on GADH can be described by Eqs. 8-10:

$\alpha-\mathrm{D}-$ galactose $+\mathrm{NADP}^{+} \mathrm{GADH} \quad \alpha-\mathrm{D}-$ galacto-1,5-lactone $+\mathrm{NADPH}$

$2 \mathrm{NAPDH}+\mathrm{OsQ} \rightarrow 2 \mathrm{NADP}^{+}+\mathrm{OsQH}_{2}$

$\mathrm{OsQH}_{2} \rightarrow \mathrm{OsQ}+2 \mathrm{H}^{+}+2 \mathrm{e}^{-}$

When the biosensor was used with a FIA system to analyze galactose, the linear range was found to be from $1.0 \mathrm{mM}$ to $3.0 \mathrm{mM}$ with a limit of detection of $0.2 \mathrm{mM}$ and precision values of $12.0-15.0 \%$; these analytical performance characteristics indicated that the GADH catalysis of galactose using osphendione as a redox mediator was successful.

\section{Conclusion}

This review has highlighted some of the novel approaches that have been employed for fabricating amperometric galactose biosensors. The analytical performance characteristics of these galactose biosensors are summarized in Table 1.
The use of GalOx offers an additional advantage over galactose dehydrogenase $(\mathrm{GADH})$, as the latter requires cofactors to be co-immobilized onto the underlying transducer. In addition to this, the response times are generally shorter for GalOx based biosensors. Consequently, only one paper reported the use of GADH in its design. However, there are several drawbacks associated with the use of GalOx-based biosensors. Significantly higher applied potentials must be used in order to generate an electrochemical response from the enzyme generated $\mathrm{H}_{2} \mathrm{O}_{2}$.

Expensive electrode materials such as graphene, gold, platinum and carbon nanotubes are often used to develop galactose biosensors employing GalOx. However, three sensors employing GalOx immobilized onto screen-printed carbon electrodes (SPCEs) as the electrode material have been described. SPCEs offer an inexpensive method for fabricating galactose biosensors, which is crucial in the commercialization of such devices for point-of-care applications. Finally, there is a plenitude of GalOx from different sources; thus, making it relatively less expensive. Cost is a relevant point to be considered for commercialization of these devices.

Acknowledgements This work was supported by funds from a World Bank African Centers of Excellence grant (ACE02-WACCBIP: Awandare) and a DELTAS Africa grant (DEL-15-007: Awandare). Francis Krampa was supported by a WACCBIP-World Bank ACE PhD fellowship and Yaw Aniweh was supported by a DELTAS Africa postdoctoral fellowship. The DELTAS Africa Initiative is an independent funding scheme of the African Academy of Sciences (AAS)'s Alliance for Accelerating Excellence in Science in Africa (AESA) and supported by the New Partnership for Africa's Development Planning and Coordinating Agency (NEPAD Agency) with funding from the Wellcome Trust (107755/Z/15/Z: Awandare) and the UK government. The views expressed in this publication are those of the author(s) and not necessarily those of AAS, NEPAD Agency, Wellcome Trust or the UK government.

Compliance with ethical standards The author(s) declare that they have no competing interests.

Open Access This article is distributed under the terms of the Creative Commons Attribution 4.0 International License (http:// creativecommons.org/licenses/by/4.0/), which permits unrestricted use, distribution, and reproduction in any medium, provided you give appropriate credit to the original author(s) and the source, provide a link to the Creative Commons license, and indicate if changes were made.

\section{References}

1. Tkáč J, Gemeiner P, Šturdik E (1999) Rapid and sensitive galactose oxidase-peroxidase biosensor for galactose detection with prolonged stability. Biotechnol Tech 13:931-936

2. Kanyong P, Pemberton RM, Jackson SK, Hart JP (2013) Development of an amperometric screen-printed galactose biosensor for serum analysis. Anal Biochem 435:114-119 
3. Kanyong P, Pemberton RM, Jackson SK, Hart JP (2016) Amperometric screen-printed galactose biosensor for cell toxicity applications. Anal Lett 49:236-244

4. Tkac J, Vostiar I, Gemeiner P, Sturdik E (2002) Indirect evidence of electron communication between the active site of galactose oxidase and a graphite electrode. Bioelectrochemistry 56:23-25

5. Baron AJ, Stevens C, Wilmot C, Seneviratnes KD, Blakeley V, Dooley DM, Phillips SEV, Knowles PF, McPherson MJ (1994) Structure and mechanism of galactose oxidase. J Biol Chem 269: 250095-225105

6. Kanyong P, Rawlinson S, Davis J (2016) A non-enzymatic sensor based on the redox of ferrocene carboxylic acid on ionic liquid filmmodified screen-printed graphite electrode for the analysis of hydrogen peroxide residues in milk. J Electroanal Chem 766:147-151

7. Xu J, Wang Y, Hu S (2017) Nanocomposites of graphene and graphene oxides: synthesis, molecular functionalization and application in electrochemical sensors and biosensors. Microchim Acta 184:1-44

8. Taylor PJ, Kmetec E, Johnson JM (1977) Design, construction and applications of a galactose selective electrode. Anal Chem 49:789794

9. Manotwitz P, Stoecker PW, Yacynych AM (1995) Galactose biosensors using composite polymers to prevent interferences. Biosens Bioelectron 10:359-370

10. Wang Y, Zhu J, Zhu R, Zhu Z, Lai Z, Chen Z (2003) Chitosan/ Prussian blue-based biosensors. Meas Sci Technol 14:831-836

11. Dalkiran B, Erden PE, Kilic E (2016) Electrochemical biosensing of galactose based on carbon materials: graphene versus multiwalled carbon nanotubes. Anal Bioanal Chem 408:4329-4339

12. Buffone GJ, Johnson JM, Lewis SA, Sparks JW (1980) Evaluation of an immobilized-enzyme analyzer for measuring galactose in serum. Clin Chem 26:339-340

13. Ji XF, Zhang YH (1993) A galactose sensor based on the platinized glassy carbon electrode modified with Nafion film. J Anal Chem 5: 519-522

14. Miyata K, Fujiwara M, Motonaka J, Moriga T, Nakabayaski I (1995) Microenzyme sensor with osmium complex and porous carbon for measuring galactose. Bull Chem Soc Jpn 68:1921-1927

15. Mannino S, Cosio MS, Buratti S (1999) Simultaneous determination of glucose and galactose in dairy products by two parallel amperometric biosensors. Ital J Food Sci 11:57-65

16. Jia NQ, Zhang ZR, Zhu JZ, Zhang GX (2003) A galactose biosensor based on the microfabricated thin film electrode. Anal Lett 36: 2095-2106

17. Sharma SK, Suman PCS, Sehgal N, Kumar A (2006) Galactose sensor based on galactose oxidase immobilized in polyvinyl formal. Sensors Actuators B Chem 119:15-19

18. Kan J, Chen C, Jing G (2005) The biosensor based on microporous polyacrylonitrile. Biocatalysis Biotransformation 23:439-444

19. Bertrand C, Coulet PR, Gautheron DC (1981) Multipurpose electrode with different enzyme systems bound to collagen films. Anal Chim Acta 126:23-34
20. Lundback H, Olsson B (1985) Amperometric determination of galactose, lactose and dihydroxyacetone using galactose oxidase in a flow injection system with immobilized enzyme reactors and online dialysis. Anal Lett 18:871-889

21. Peteu SF, Emerson D, Worden RM (1996) A clark-type oxidase enzyme-based amperometric microbiosensor for sensing glucose, galactose or choline. Biosens Bioelectron 11:1059-1071

22. Sharma SK, Singhal R, Malhotra BD, Sehgal N, Kumar A (2004) Langmuir-Blodgett film based biosensors for estimation of galactose in milk. Electrohim Acta 49:2479-2485

23. Sung WJ, Bae YH (2006) Glucose oxidase, lactate oxidase and galactose oxidase enzyme electrode based on polypyrrole with polyanion/PEG/enzyme conjugate dopant. Sensors Actuators B Chem 114:164-169

24. Tkáč J, Whittaker JW, Ruzgas T (2007) The use of single walled carbon nanotubes dispersed in a chitosan matrix for preparation of a galactose biosensor. Biosens Bioelectron 22:1820-1824

25. Cevik E, Senel M, Abasiyanik MF (2010) Construction of biosensor for determination of galactose with galactose oxidase immobilized on polymeric mediator contains ferrocene. Curr Appl Phys 10:1313-1316

26. Senel M, Bozgeyik I, Cevik E, Abasiyanik MF (2011) A novel amperometric galactose biosensor based on galactose oxidasepoly(N-glycidylpyrrole-co-pyrrole). Synth Met 161:440-444

27. Lee KN, Lee Y, Son Y (2011) Enhanced sensitivity of a galactose biosensor fabricated with a bundle of conducting polymer microtubules. Electroanalysis 23:2125-2130

28. Coche-Guerente L, Cosnier S, Innocent C, Mailley P (1995) Development of amperometric biosensors based on the immobilization of enzymes in polymer films electrogenerated from a series of amphiphilic pyrrole derivatives. Anal Chim Acta 311:23-30

29. Yang Y, Mu S, Chen H (1998) Electrochemical synthesis of polypyrrole for the immobilization of galactose oxidase. Synth Met 92: $173-178$

30. Gülce H, Ataman I, Gülce A, Yildiz A (2002) A new amperometric enzyme electrode for galactose determination. Enzym Microb Technol 30:42-44

31. Brahim SI, Maharaj D, Narinesingh D, Guiseppi-Elie A (2002) Design and characterization of a galactose biosensor using a novel polypyrrole-hydrogel composite membrane. Anal Lett 35:797-812

32. Ekinci E, Pasahan A (2004) Poly(4-methyoxyphenol) film as a galactose-sensing material. Eur Polym J 40:1605-1608

33. Charmantray F, Touisni N, Hecquet L, Mousty C (2013) Amperometric biosensor based on galactose oxidase immobilized in clay matrix. Electroanalysis 25:630-635

34. Charmantray F, Touisni N, Hecquet L, Noguer T, Mousty C (2015) Galactose oxidase/Prussian blue based biosensors. Electroanalysis 27:1341-1344

35. Maestre E, Katakis I, Narváez A, Dominguez E (2005) A multianalyte flow electrochemical cell: application to the simultaneous determination of carbohydrates based on bioelectrocatalytic detection. Biosens Bioelectron 21:774-781 